\title{
Forward-Running Detonation Drivers for High-Enthalpy Shock Tunnels
}

\author{
Z. L. Jiang, ${ }^{*} \mathrm{~W}$. Zhao, ${ }^{\dagger}$ and C. Wang \\ Chinese Academy of Sciences, 100080 Beijing, People's Republic of China \\ and \\ K. Takayama ${ }^{\S}$ \\ Tohoku University, Sendai 980-8577, Japan
}

\begin{abstract}
To improve the quality of driving flows generated with detonation-driven shock tunnels operated in the forwardrunning mode, various detonation drivers with specially designed sections were examined. Four configurations of the specially designed section, three with different converging angles and one with a cavity ring, were simulated by solving the Euler equations implemented with a pseudo kinetic reaction model. From the first three cases, it is observed that the reflection of detonation fronts at the converging wall results in an upstream-traveling shock wave that can increase the flow pressure that has decreased due to expansion waves, which leads to improvement of the driving flow. The configuration with a cavity ring is found to be more promising because the upstream-traveling shock wave appears stronger and the detonation front is less overdriven. Although pressure fluctuations due to shock wave focusing and shock wave reflection are observable in these detonation-drivers, they attenuate very rapidly to an acceptable level as the detonation wave propagates downstream. Based on the numerical observations, a new detonation-driven shock tunnel with a cavity ring is designed and installed for experimental investigation. Experimental results confirm the conclusion drawn from numerical simulations. The generated driving flow in this shock tunnel could maintain uniformity for as long as $4 \mathrm{~ms}$. Feasibility of the proposed detonation driver for high-enthalpy shock tunnels is well demonstrated.
\end{abstract}

\section{Nomenclature}

$\begin{array}{ll}D_{\mathrm{CJ}} & =\text { Chapman-Jouguet }(\mathrm{C}-\mathrm{J}) \text { detonation velocity } \\ e & =\text { total energy per mass } \\ \boldsymbol{F} & =\text { flux in } x \text { direction } \\ \boldsymbol{G} & =\text { flux in } \gamma \text { direction } \\ h & =\text { chemical reaction rate } \\ m_{1} & =\text { molecular weight of the reactant } \\ m_{2} & =\text { molecular weight of the product } \\ P_{\mathrm{CJ}} & =\text { C-J detonation pressure } \\ P_{i} & =\text { initial pressure in driver section } \\ P_{\text {ignit }} & =\text { ignition pressure } \\ P_{0} & =\text { initial pressure in driven section } \\ p & =\text { pressure } \\ Q & =\text { reaction heat per mass } \\ S, \boldsymbol{H} & =\text { source terms } \\ T_{i} & =\text { initial temperature in driver section } \\ \boldsymbol{U} & =\text { state variable } \\ u & =\text { velocity component in } x \text { direction } \\ v & =\text { velocity component in } y \text { direction } \\ Z_{1} & =\text { mass fraction of the reactant } \\ Z_{2} & =\text { mass fraction of the product } \\ \alpha, \beta, A \text { det } & =\text { tuned constants of reaction model } \\ \gamma & =\text { effective adiabatic exponent } \\ \gamma_{1} & =\text { adiabatic exponent of the reactant } \\ \gamma_{2} & =\text { adiabatic exponent of the product } \\ \Delta s & =\text { local mesh size }\end{array}$

Received 31 March 2001; revision received 29 March 2002; accepted for publication 28 May 2002. Copyright (C) 2002 by the American Institute of Aeronautics and Astronautics, Inc. All rights reserved. Copies of this paper may be made for personal or internal use, on condition that the copier pay the $\$ 10.00$ per-copy fee to the Copyright Clearance Center, Inc., 222 Rosewood Drive, Danvers, MA 01923; include the code 0001-1452/02 \$10.00 in correspondence with the CCC.

*Professor, Key Laboratory of High Temperature Gasdynamics, Institute of Mechanics; zljiang @imech.ac.cn.

${ }^{\dagger}$ Associate Professor, Key Laboratory of High Temperature Gasdynamics, Institute of Mechanics; zw@imech.ac.cn.

*Doctoral Candidate, Key Laboratory of High Temperature Gasdynamics, Institute of Mechanics; cwang@imech.ac.cn.

${ }^{\S}$ Professor, Shock Wave Research Center, Institute of Fluid Science, 2-1-1 Katahira, Aoba-ku; takayama@ ifs.tohoku.ac.jp. Member AIAA.

$\begin{array}{ll}\Delta x & =\text { mesh size in } x \text { direction } \\ \Delta \gamma & =\text { mesh size in } \gamma \text { direction } \\ \rho & =\text { density } \\ \rho_{1} & =\text { reactant density } \\ \rho_{2} & =\text { product density }\end{array}$

\section{Introduction}

$\mathbf{F}$ ROM the beginning of the past century, aerodynamic research has been driven by the urge to fly faster and higher. So far, supersonic flight has been achieved, and the new century is expected to be the era of practical hypersonic flight. The urge poses many exciting challenges to aerodynamic scientists and engineers. The development of high-enthalpy shock tunnels for reliable ground testing of hypersonic vehicles is one of these research topics. This is because, for flight speeds higher than Mach 8, that is, at high stagnation enthalpies, there exist technological barriers that would be very hard to overcome with the blowdown-typehypersonic wind tunnels. Therefore, shock tunnels become the facilities of choice for high-enthalpy ground testing. So far as their operation concepts are concerned, two major kinds of the shock tunnels are widely applied to generate high-enthalpy test flows: One is driven by a free piston and the other by gaseous detonation.

The concept of the free-piston driver was studied as early as 1959 at the National Research Council in Ottawa, Canada, and the work was reported later by Stalker ${ }^{1}$ in 1961. A series of free-piston shock tunnels was then initiated in Australia, and the first such facility was realized by modifying a conventional shock tunnel. ${ }^{2}$ Within the next three decades, many free-piston-drivenshock tunnels have been constructed and installed all over the world, such as T2 and T3 at the Australian National University, Australia; T4 at the University of Queensland, Australia; T5 at the California Institute of Technology, United States; High Enthalpy Shock Tunnel Göttingen at Göttingen, Germany; and High Enthalpy Shock Tunnel Kakuda and the HighEnthalpy Shock Tunnel at the National Aerospace Laboratory at Kakuda, Japan. ${ }^{3-8}$ These shock tunnels are widely used to generate high-enthalpy test flows with effective test time ranging from 0.1 to $2.0 \mathrm{~ms}$

An alternate technique is the so-called detonation driver in which the driver gas is generated by the detonation of a gaseous reactive 
mixture. As early as 1957, Bird $^{9}$ studied the wave processes in the detonation driver and its superior performance compared to the conventional shock driver. Since then, several detonation-driven shock tunnels have been constructed and put into operation. Yu developed a detonation-drivenshock tube in the backward running mode at the Institute of Mechanics in Beijing ${ }^{10,11}$ and later modified it to be a shock tunnel. ${ }^{12}$ Mean while, a 140 -mm-diam shock tunnel was constructed at the the Shock Wave Laboratory, Rheinisch Westfälischen Techischen Hochschule Aachen, Germany, which was largely performed as a joint research project with the Institute of Mechanics, Chinese Academy of Sciences. ${ }^{13}$ In addition, two detonation-driven shock tunnels configured in the forward-running mode are in operation at General Applied Science Laboratories in New York and at the University of Texas in Arlington. ${ }^{14}$ The effective test time achieved with the detonation-driven shock tunnels can be as long as $4.0 \mathrm{~ms}$.

When the detonation-driven shock tunnels are compared with free-piston-driventunnel, one could see that the detonation-driven shock tunnel is easier to operate with a relatively longer test time and lower operation cost. However, before completing this technology, the problems related to the fundamental physics of detonation and the assessment of its applicationto high-enthalpyshock tunnels have to be investigated.

To explain the operational concept of detonation drivers, it is helpful to recall the Chapman ${ }^{15}-J_{\text {Jouguet }}{ }^{16}$ theory (C-J theory) and the Taylor similarity law, ${ }^{17}$ which have proved to describe well the global features of gaseous detonation. Assume that a tube closed at one end is filled with a detonable gas mixture at rest and that the detonation is initiated at its closed end. The wave motion can be described by the $\mathrm{C}-\mathrm{J}$ theory and the Taylor similarity law. In this wave system, the detonation front is taken as a shock wave propagating into the unburned detonable gas mixture, which compresses the gas mixture ahead of it and elevates the gas pressure and temperature so high as to initiate an auto-ignition. Hence, a chemical reaction concurrently takes place in an infinitely thin reaction zone, and then the burned gas reaches the state that can be determined with the $\mathrm{C}-\mathrm{J}$ condition that states that, behind the reaction zone, there is a plane in which the velocity of the gas particles of the detonation product relative to the wave front is equal to the local speed of sound. Behind this $\mathbf{C}-\mathrm{J}$ plane, the flow expands to a steady state at zero velocity, and the uniform pressure is about $40 \%$ of the $\mathrm{C}-\mathrm{J}$ pressure. The length of the flow in the uniform state is about half of the distance in which the detonation wave has propagated. The distributions of detonation wave properties along the length of the detonation tube are schematically presented in Fig. 1, which will be used to discuss further the operating concept of detonation-driven shock tunnels.

If a driven section is connected to the left of the detonation tube, as shown in Fig. 1, the tail of the Taylor expansion wave is utilized as the high-pressure driver gas. ${ }^{12,13}$ This operational concept is referred to as the backward-running mode because the detonation front propagates upstream, instead of in the direction of the flow in the driven (or shock) tube. A uniform driving flow is obtainable in this mode, but the driving pressure and temperature so far achievable is not as high as that created with a heavy free-piston compression because of the pressure limitation of the detonation tube materials. From the detonation properties presented in Fig. 1, it is known that the driver gas pressure is only about $40 \%$ of the $\mathrm{C}-\mathrm{J}$ pressure and that the kinetic energy of the gas flow is zero. This leads to another way to drive the test gas in the driven section, by connecting the

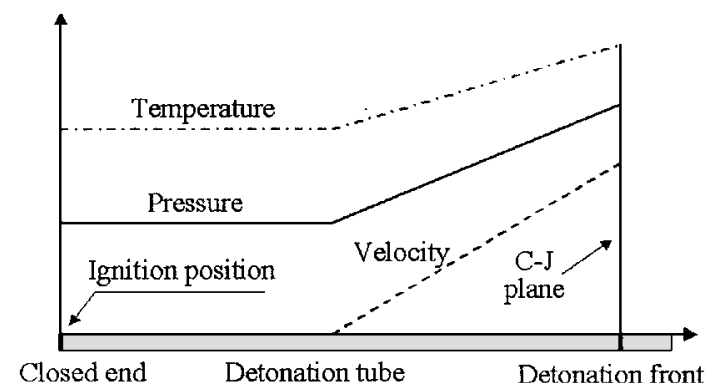

Fig. 1 Distributions of detonation wave properties along the length of a detonation tube; the problem described with the Taylor similarity law. ${ }^{17}$

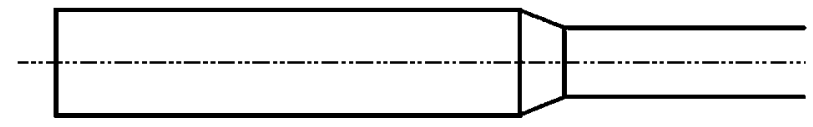

a)

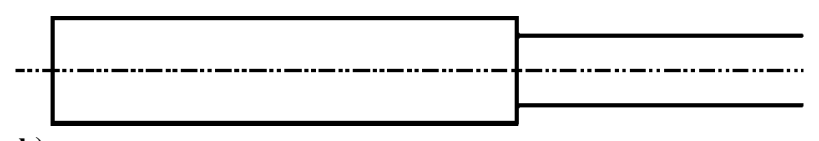

b)

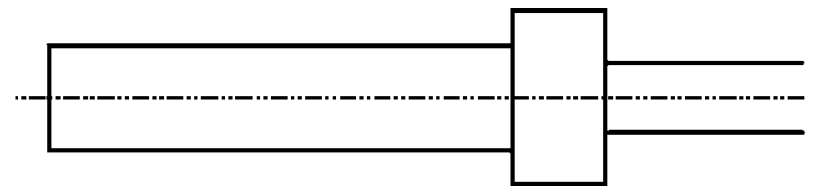

c)

Fig. 2 Schematic configurations of detonation drivers proposed for numerical investigation.

driven section to the right of the detonation tube; this mode of operation is called the forward-running mode. That is, the detonation propagates forward in the direction of the primary shock propagation in the driven section. It is obvious that a driving flow of much higher stagnation pressure could be obtainable, but it is not very uniform due to the Taylor expansion behind the detonation front.

To overcome the deficiency of the detonation driver operated in the forward-running model, several detonation drivers with a specially designed section are proposed in this paper. The basic idea is to add this section to the conventional detonation driver so that an upstream-traveling shock wave could be generated to increase the flow pressure that has decreased due to expansion waves. Three types of detonation drivers, as shown in Fig. 2, are numerically investigated to examine the driving flow uniformity. The first type of detonation driver consists of a large diameter tube, a small diameter tube, and a converging section between them, as shown in Fig. 2a. Two configurations are obtained by varying the convergence angle from 30 to $45 \mathrm{deg}$. The second type of detonationdriver, as shown in Fig. $2 b$, is similar to the first one but without the converging section. Actually, this type can be achieved by increasing the convergence angle of the first type to $90 \mathrm{deg}$. In the third type of detonationdriver, as shown in Fig. 2c, the converging section is replaced by a cavity ring.

When the Euler equations are solved for a binary mixture of perfect gases implemented with a pseudokinetic reaction model ${ }^{18}$ numerical simulations for the four cases are carried out to investigate the effects of various configurations on the driving flow uniformity. It is found that the detonation drivers with converging sections do improve the driving flow uniformity, but the one with a cavity ring shows more promising character. Following the numerical investigation, a detonation-driven shock tunnel with a cavity ring was manufactured and installed at the Institute of Mechanics, Chinese Academy of Sciences, Beijing. Some experiments were conducted, and pressure histories (pressure variations against time) were measured at the end wall of the driven section. The experimental data confirm the numerical investigation, which shows that multiple shock reflections resulting from the specially designed section lead to the generation of an upstream-travelingshock wave that can increase the flow pressure behind the detonation front and help to create a uniform test gas slug.

\section{Governing Equations and Numerical Methods}

The flow phenomena that are the concern of this paper are the wave dynamic processes in which viscosity effects are negligible. The two-dimensional Euler equations in conservation form for a binary mixture of the perfect gases are written in cylindrical coordinates as

$$
\frac{\partial \boldsymbol{U}}{\partial t}+\frac{\partial \boldsymbol{F}}{\partial x}+\frac{\partial \boldsymbol{G}}{\partial r}+\frac{1}{r} \boldsymbol{S}=\boldsymbol{H}
$$

where $x, r$, and $t$ are the coordinates and time. $\boldsymbol{U}, \boldsymbol{F}, \boldsymbol{G}, \boldsymbol{S}$, and $\boldsymbol{H}$ are the state variables, fluxes, and sources, respectively, and are given 
by

$$
\begin{gathered}
\boldsymbol{U}=\left(\begin{array}{c}
\rho_{1} \\
\rho_{2} \\
\rho u \\
\rho v \\
e
\end{array}\right), \quad \boldsymbol{F}=\left(\begin{array}{c}
\rho_{1} u \\
\rho_{2} u \\
\rho u^{2}+p \\
\rho u v \\
(e+p) u
\end{array}\right), \quad \boldsymbol{G}=\left(\begin{array}{c}
\rho_{1} v \\
\rho_{2} v \\
\rho u \\
\rho v^{2}+ \\
(e+p)
\end{array}\right. \\
\boldsymbol{S}=\left(\begin{array}{c}
\rho_{1} v \\
\rho_{2} v \\
\rho u v \\
\rho v^{2} \\
(e+p) v
\end{array}\right), \quad \boldsymbol{H}=\left(\begin{array}{c}
\rho h\left(\rho_{1}, \rho_{2}, p\right) \\
-\rho h\left(\rho_{1}, \rho_{2}, p\right) \\
0 \\
0 \\
0
\end{array}\right)
\end{gathered}
$$$$
\boldsymbol{G}=\left(\begin{array}{c}
\rho_{1} v \\
\rho_{2} v \\
\rho u v \\
\rho v^{2}+p \\
(e+p) v
\end{array}\right)
$$

where the primitive variables in the unknown $\boldsymbol{U}$ are density $\rho$; the reactant density $\rho_{1}$; the product density $\rho_{2}$; velocity components $u$ and $v$ in the $x$ or $\gamma$ direction, respectively; the reaction rate $h\left(\rho_{1}, \rho_{2}, p\right)$, and total energy per unit mass $e$, related to the equation of state for the perfect gaseous mixture by

$$
e=p /(\gamma-1)+\frac{1}{2} \rho\left(u^{2}+v^{2}\right)+\rho_{1} Q
$$

where $Q$ is the chemical reaction heat release per unit mass. It is assumed that the equation of state is independent of the composition of gas mixtures, but the internal specific energy is modified to include the latent heat of combustion. The density of the gas mixture is calculated by

$$
\rho=\rho_{1}+\rho_{2}
$$

If mass fractions are denoted by $Z_{1}=\rho_{1} / \rho$ and $Z_{2}=\rho_{2} / \rho$, the effective adiabatic exponent of the gas mixture, $\gamma$, can be put in the form

$$
\gamma=\frac{Z_{1} \gamma_{1} / m_{1}\left(\gamma_{1}-1\right)+Z_{2} \gamma_{2} / m_{2}\left(\gamma_{2}-1\right)}{Z_{1} / m_{1}\left(\gamma_{1}-1\right)+Z_{2} / m_{2}\left(\gamma_{2}-1\right)}
$$

where $\gamma_{1}$ and $m_{1}$ are the adiabatic exponentand molecular weight for the reactant and $\gamma_{2}$ and $m_{2}$ are the respectiveparameters for the product. The governing equations (1) are discretized using a dispersioncontrolled scheme proposed by Jiang et al. ${ }^{19}$ Time-marching integration is performed using a Runge-Kutta integration method of second-order accuracy. The computational mesh uses an equally spaced Cartesian grid to accommodate the oblique rigid wall, where the ratio of mesh size $\Delta \gamma / \Delta x$ is chosen so that the oblique wall could coincide with the cell diagonal. This choice enables a simple and accurate algorithm to be implemented with our scheme, producing a rigid-wall boundary condition of second-order accuracy through computing the mirror-image flow states at virtual grid points outside the wall. A $5000 \times 30$ mesh system is generated to cover the detonation driver of $5 \mathrm{~m}$ in length and $60 \mathrm{~mm}$ in diameter. The mesh size is $1 \mathrm{~mm}$ wide, and its length is adjustable according to the given convergence angle.

\section{Reaction Model}

To examine gasdynamic characteristicsof a detonationdriver, the entire detonation tube has to be simulated. The mesh size that could be used, as mentioned in the last section, is still too large to capture accurately either the detonation structure or the thickness of detonation front because of the large length-to-diameter ratio. Therefore, the strategy for selecting a kinetic reaction rate model is to obtain an idealized the Taylor expansion wave having an infinitely thin reaction zone. The emphasis here is to predict the gasdynamics in front of and following a detonation front, rather than to produce correctly either the detailed detonation structure or the process by which a shock wave propagating in the reactive gas mixture evolves gradually into a detonation wave. The following pseudokinetic reaction rate $^{18}$ has been proved to be acceptable for our purposes:

$$
h\left(\rho_{1}, \rho_{2}, p\right)= \begin{cases}0 & p<P_{\text {ignit }} \\ -A \operatorname{det}\left(D_{\mathrm{CJ}} / \Delta s\right)\left(p / P_{\mathrm{CJ}}\right)^{\alpha} Z_{1}^{\beta} & \text { otherwise }\end{cases}
$$

where $A$ det, $\alpha$, and $\beta$ are dimensionless constants of order unity whose values are determined by inspecting one-dimensional simulations of detonation and looking for the captured solutions close to the Taylor similar solutions and the $\mathrm{C}-\mathrm{J}$ conditions, which are known to predict well the global features of gaseous detonation. A set of values for these constants was determined by such a procedure and is given next. ${ }^{18}$ These constants depend mainly on the gas mixture and vary slightly with numerical schemes. Once tuned they keep constant throughout computation:

$$
P_{\text {ignit }}=0.4 P_{\mathrm{CJ}}, \quad \alpha=1.0, \quad \beta=0.5, \quad A \operatorname{det}=1.6
$$

All of the computational results presented in this paper have been produced with this set of tuned constants of the pseudokinetic reaction model. Validation of calculated results with this model has been carried out by comparison with experimental data. The maximum discrepancy in the $\mathrm{C}-\mathrm{J}$ velocity, pressure, and Mach number is less than 5\% (Ref. 18), which is an acceptable level for our purpose of analyzing the wave dynamic processes. However, although this reaction model is capable of describing the flow features as presented by the Taylor similarity law, the von Neumann spike (or shock pressure) can not be captured, even if the mesh sizes were sufficiently fine. A more detailed reaction model could have been used to simulate the detonation structure and its associated shock pressure, but it would also consume much of the computational resources. Although the current model does not include the reaction zone in the detonation, it nevertheless contains the essential qualitative features of the propagating wave and is still a useful tool for the current parametric study.

\section{Numerical Results and Discussion}

With the use of the governing equations, the numerical methods, and the chemical reaction model introduced in the preceding sections, the three types of the detonation drivers (Fig. 2) are simulated to examine the wave interaction in the specially designed section and its effects on the driving flow. In these test cases, the detonation driver is about $5 \mathrm{~m}$ long in total and consists of three sections: two straight tubes with different diameters and a specially designed areachanging section between the two tubes. The detonative gas used in numerical simulations is a mixture of $2 \mathrm{H}_{2}+\mathrm{O}_{2}$ at the initial condition of $P_{i}=0.1 \mathrm{MPa}$ and $T_{i}=298.15 \mathrm{~K}$. The adiabatic exponents are taken to be 1.402 for the reactants and 1.219 for the products. The reaction energy is $Q=8.39 \times 10^{6} \mathrm{~J} / \mathrm{kg}$. The molecular weights are 12 for the reactants and 18 for the products.

In the following discussion, two major physical processes are emphasized. The first process is the wave interaction after the detonation front moves into the specially designed section. Sequential isobars of the flow motion near the specially designed section will be presented to show the wave propagation, reflection, and focusing. The other is the pressure variations induced by the wave interaction on the driving flow. Sequential profiles of pressure distributions along the axis of symmetry in the detonation drivers are plotted for investigation. From these results, the observed wave phenomena are explained, the mechanism behind the phenomena is explored, and their effects on driving flows are discussed.

\section{A. Detonation Driver with 30-Degree Convergence Angle}

The first case carried out is the configuration of type A as shown in Fig. 2a by setting a 30-deg convergence angle. For this case, the large straight tube is $3.5 \mathrm{~m}$ long and $90 \mathrm{~mm}$ in diameter, and the small tube is $1.5 \mathrm{~m}$ long and $60 \mathrm{~mm}$ in diameter. Between these two tubes, a section with a 30-deg convergence angle is inserted. Numerical results are plotted in Figs. 3 and 4.

Figure 3 shows four time sequences of isobars of the flow motion shortly before and after the detonation front moves into the converging section. Time interval is $25 \mu$ s between Figs. $3 \mathrm{a}, 3 \mathrm{~b}$, and $3 \mathrm{c}$, and $50 \mu$ s between Figs. $3 \mathrm{c}$ and $3 \mathrm{~d}$. First, a planar detonation wave, that is, the front of the Taylor expansion wave, as observed in Fig. 3a, arrives at the entrance of the converging section, behind which expansion waves are observable from the isobar as annotated. The detonation wave front reflects from the converging wall in Fig. 3b, and 
Mach reflections develop later. Wave diffraction is also observable behind the Mach stem as the expansion waves behind the detonation front pass through the converging section. Then, the reflected shock wave coalesces (Fig. 3c), and a nearly normal shock wave develops and propagates upstream, as shown in Fig. 3d. This shock wave can elevate both the pressure and temperature of the flow that has passed through the shock wave. At the same time, a downstream-traveling shock wave is also generated and catches up with the detonation front very quickly as shown in Fig. 3c, which will result in an overdriven detonation front. Moreover, it is observable in Figs. $3 \mathrm{c}$ and $3 \mathrm{~d}$ that transverse wave reflections repeatedly appear between the detonation front and the upstream-travelingshock wave, which may induce pressure fluctuations in this region.

Sequential pressure distributions along the axis of symmetry of case 1 are presented in Fig. 4 at four time steps. Figure 4a corresponds to the time instant when a detonation front arrives at the entrance of the converging section, as shown in Fig. 3a. The typical pressure distribution of the Taylor expansion wave is observable here, which consists of the $\mathrm{C}-\mathrm{J}$ plane in the detonation front, the

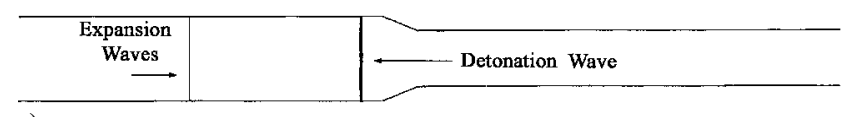

a)

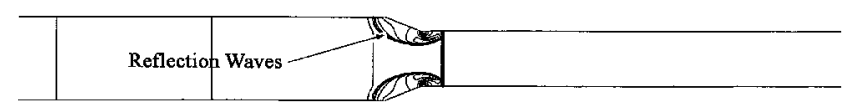

b)

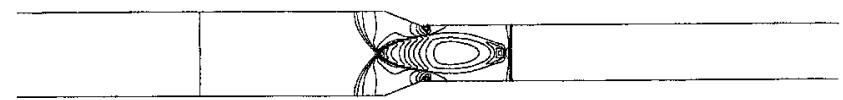

c)

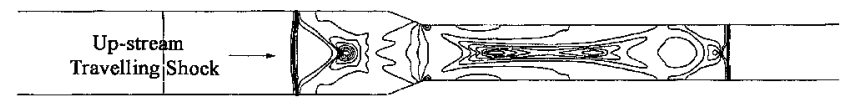

d)

Fig. 3 Time sequences of isobars of the flow motion showing wave interactions in case 1: 30 -deg converging angle. expansion waves, and a flow slug at uniform pressure. This result shows that the reaction model adopted here can represent well the Taylor similarity law. Figure $4 \mathrm{~b}$ shows the flow state shortly after the reflected shock waves merge at the axis of symmetry, as shown in Fig. 3c. Two pressure peaks appear in Fig. 4b: The higher one represents the postshock pressure of the upstream-traveling shock wave, and the other represents the detonation front. The higher peak pressure is about 40 times higher than the initial pressure but decreases very rapidly as the upstream traveling shock wave propagates forward, as seen in Figs. $4 \mathrm{c}$ and $4 \mathrm{~d}$. The pressure peak behind the detonation front is higher than the $\mathrm{C}-\mathrm{J}$ pressure (Fig. 4a), which means the detonation front is overdriven. The upstream-traveling shock wave induces a pressure level that achieved an average value of $70 \%$ of the C-J pressure, as shown in Fig. 4d. Although the pressure fluctuations shown in Fig. 4c due to the repeatedly reflected shock waves are rather higher, they attenuate quite quickly, as seen in Fig. 4d. This implies that a more uniform driving flow could be expected in the driven section. Furthermore, when the pressure profile in Fig. 4a is compared with that in Fig. 4d, it can be concluded that, as a driving flow, the flow state shown in Fig. $4 \mathrm{~d}$ is more preferable than that shown in Fig. 4a; therefore, the converging section is helpful to increase the flow pressure behind the detonation front and to produce a more uniform driving flow.

\section{B. Detonation Driver with 45-Degree Convergence Angle}

From the numericalsimulation of case 1, the driving flow is known to be improved, but the flow pressure between positions at 2.7 and $4.0 \mathrm{~m}$, as shown in Fig. 4d, is still lower than that behind the detonation front. This means that an even stronger upstream-traveling shock wave is required. Case 2 is designed by setting the convergence angle to be $45 \mathrm{deg}$ in type A, as shown in Fig. 2. The idea behind this case is that a larger reflection angle may induce a stronger upstream-traveling shock wave. Numerical results of case 2 are presented in Figs. 5 and 6.

In Figs. 5 and 6, the wave interactions so far observable appear to be similar to those shown in Figs. 3 and 4. However, some features different from case 1 are worth pointing out. The stronger shock reflection generated, as shown in Fig. 5b, results in a stronger upstream-traveling shock wave. Therefore, a higher peak pressure due to shock wave focusing is observablein the pressure distribution
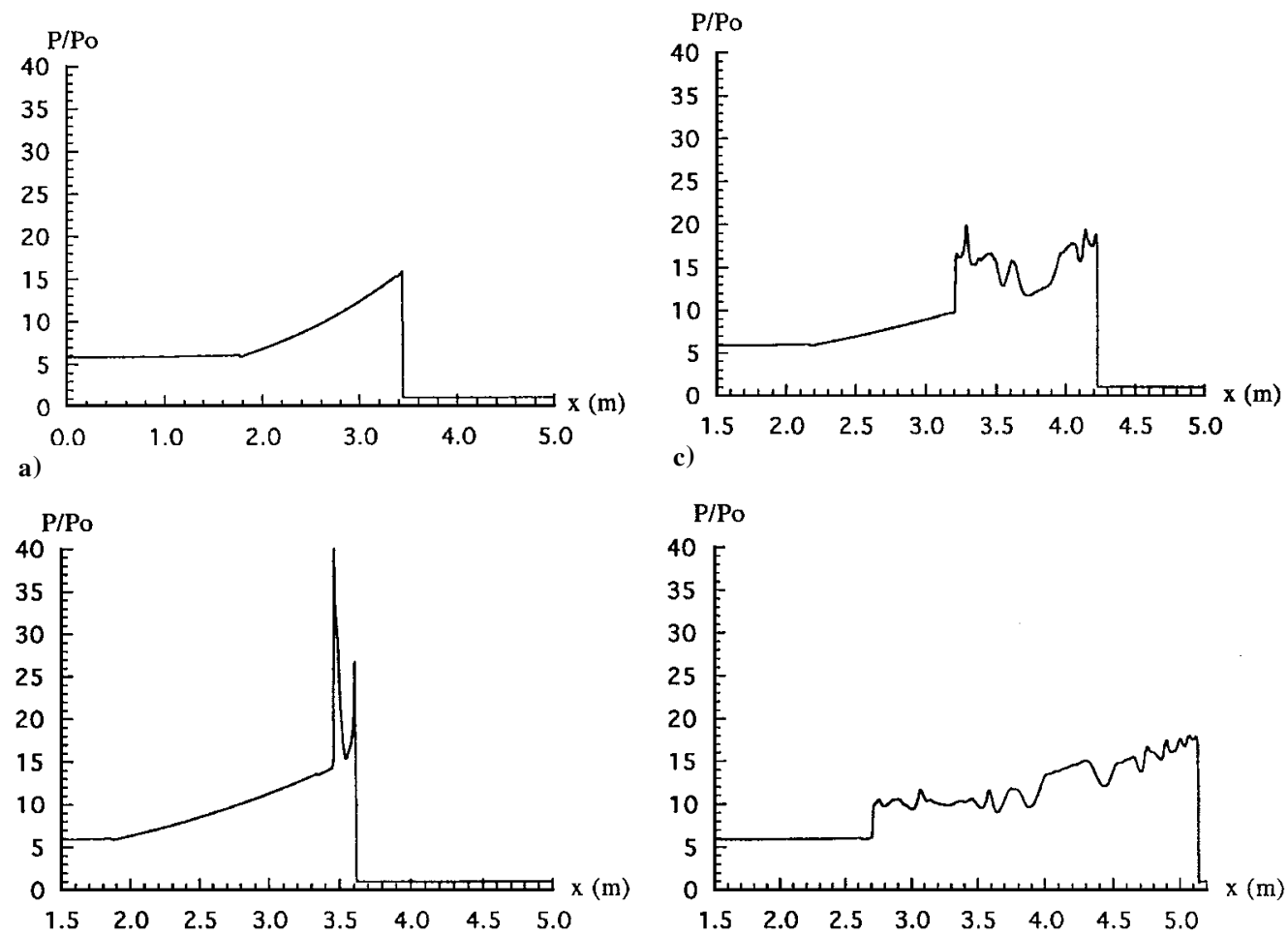

b)

d)

Fig. 4 Sequential pressure profiles along the axis of symmetry of case 1: 30-deg converging angle. 
of Fig. 6b, which is $25 \%$ higher than that shown in Fig. 4 b. The postshock pressure behind the upstream-traveling shock wave in Fig. 6d is $10 \%$ higher than that shown in Fig. 4d. This is useful for generating uniform driving flows. When the peak pressure at the detonation front in Figs. 4d and 6d is examined, the peak pressure in Fig. 6d is lower. This means the detonation front in case 2 is less overdriven. From the viewpoint of the uniformity of driving flows, note that a less overdriven-detonation front will benefit the detonation driver and produce a more uniform driving flow.

From the numerical result it is understood that the configuration of case 2 is better than case 1 . This is attributable to the larger convergence angle that can induce a stronger upstream-traveling shock wave that is favorable to the generation of a more uniform flow pressure.

\section{Detonation Driver with 90-Degree Convergence Angle}

Motivated by observations in the last two cases, the third case is designed with a 90-deg convergence angle so that a much stronger

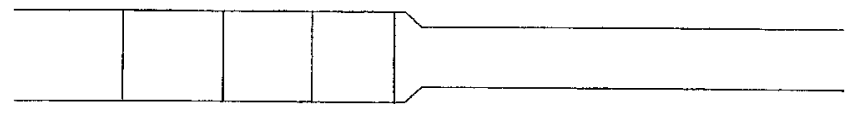

a)

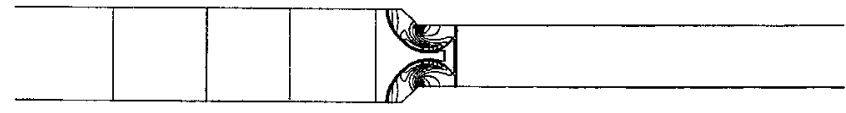

b)

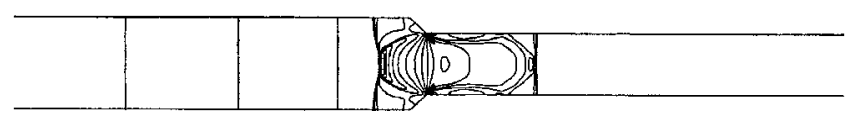

c)

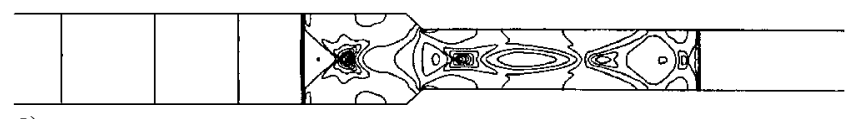

d)

Fig. 5 Time sequences of isobars of the flow motion showing wave interactions in case 2 with a $45-$ deg converging angle.

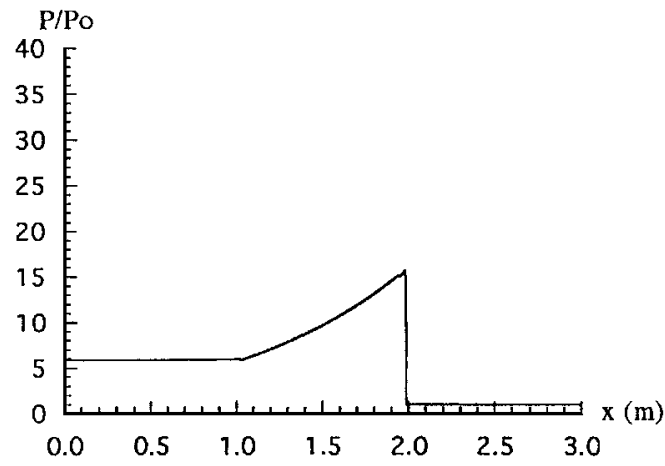

a)

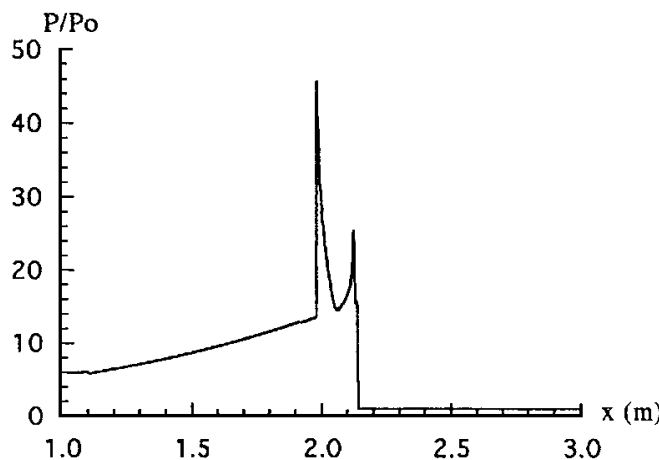

b)

Fig. 6 Sequential pressure profiles along the axis of symmetry of case 2 with a 45-deg converging angle. upstream-travelingshock wave could be obtained. The configuration of case 3 is taken to be type B, as shown in Fig. $2 b$; actually it is the same as type A with a 90-deg convergence angle. Four sequential isobars of the wave interaction near the area-changing section are presented in Fig. 7, and the sequential pressure distributions along the axis of symmetry are plotted in Fig. 8.

It would be helpful to examine the performance of the three detonation drivers together by comparing Fig. 7 with Figs. 3 and 5, as well as Fig. 8 with Figs. 4 and 6, because their configurations are similar. In these three cases, the major wave phenomenon is that the shock wave reflects from the converging wall and focuses on the axis of symmetry, which leads to the generation of two shock waves. One is the upstream-traveling shock wave as shown in Figs. 3d, 5d, and $7 \mathrm{~d}$, which increases the pressure of the gas it processes. The stronger the shock wave is, the greater the improvement on driving flows. Another shock wave is a downstream-traveling one that can catch up with the detonation front and results in an overdriven detonation front. This shock wave is difficult to observe because shock

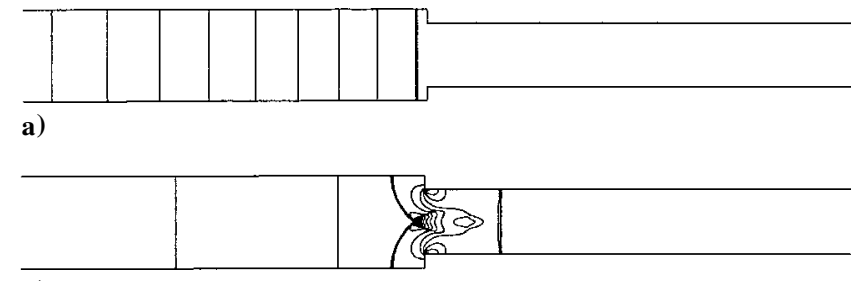

b)

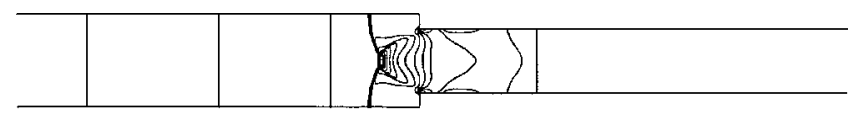

c)

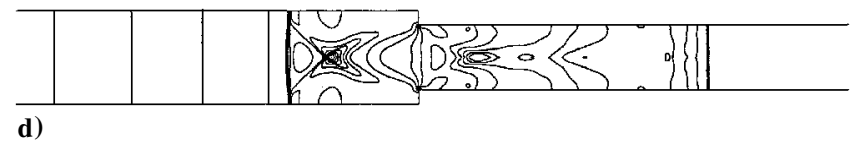

Fig. 7 Time sequences of isobars of the flow motion showing wave interactions in case 3 with a 90 -deg converging angle.
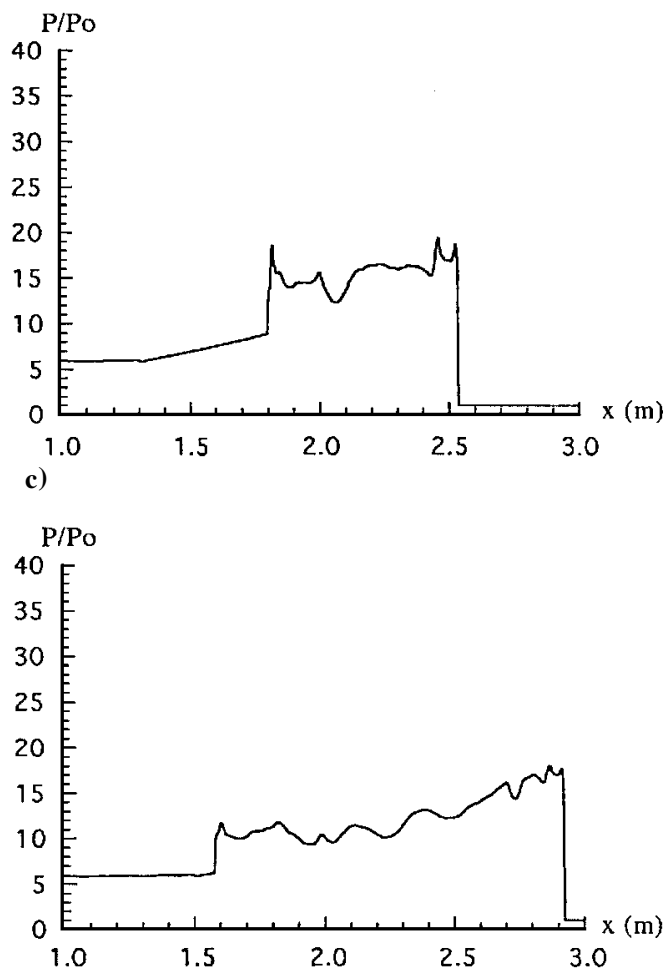

d) 

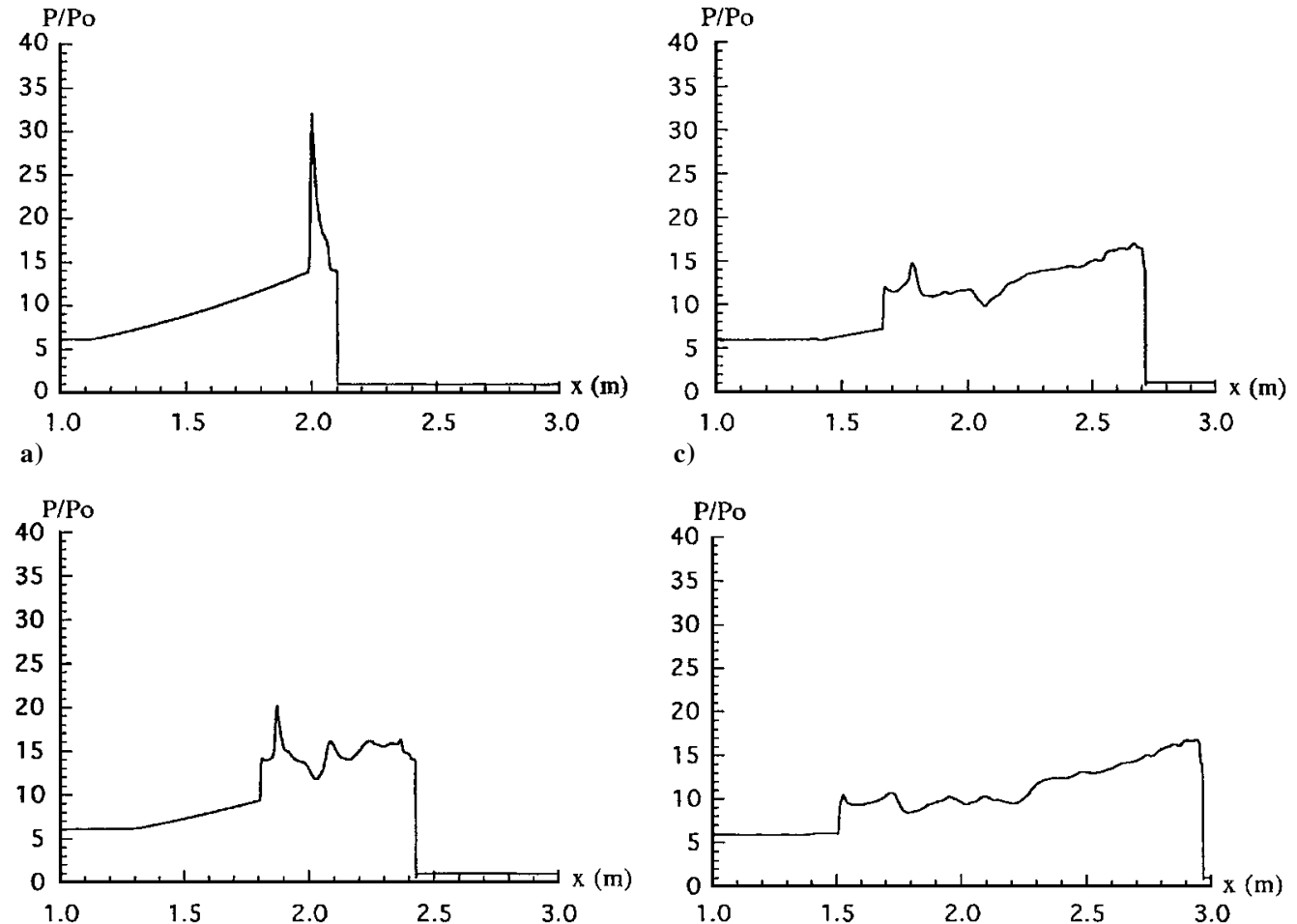

c)

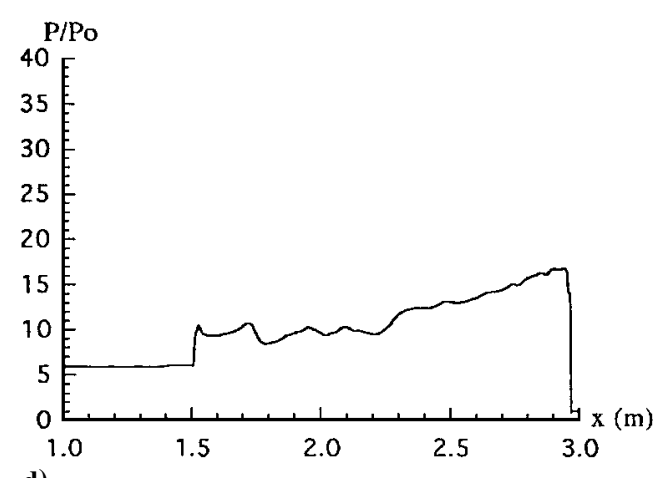

b)

Fig. 8 Sequential pressure profiles along the axis of symmetry of case 3 with a 90-deg converging angle.

wave focusing appears just shortly behind the detonation front, but it can be identified from Figs. $4 \mathrm{~b}$ and $6 \mathrm{~b}$. The overpressure behind the detonation fronts is 27 in Fig. 4b and 24 in Fig. 6b. Both are higher than the C-J pressure of 16 in Figs. $4 \mathrm{a}$ and $6 \mathrm{a}$. This results from the interaction of the downstream-traveling shock wave with the detonation fronts. The overdriven detonation front will attenuate gradually because it is not self-sustained, but the driving flow uniformity will be affected to some extent.

When the numerical results of the three test cases are examined, it is found that from case 1 to case 3 , the upstream-traveling shock wave becomes stronger and stronger and that the detonation front gets less and less overdriven. The difference between cases 1 and 2 is obvious, but it is minor between case 2 and case 3 . Moreover, the increase of the postshock pressure behind the upstream-traveling shock wave is not easy to identify from Figs. $6 \mathrm{~d}$ and $8 \mathrm{~d}$, but the detonation front in case 3 appears to be not as much overdriven as in case 2. Therefore, the pressure profile in Fig. $8 \mathrm{~d}$ appears more favorable and is achieved by avoiding the overdriven detonation front. A key mechanism in these three cases with different convergence angles is the amount of transverse shock reflections. As the angle increases, the component of the transverse reflections decreases. In the limit, as the angle becomes $90 \mathrm{deg}$, there is little or no transverse reflection, and the reflections are all axial and in the upstream direction only. This is reason that the flow state appears the cleanest and the detonation front is less overdriven in Fig. 8d.

In conclusion, the generation of a stronger upstream-traveling shock wave and a less overdriven detonation front in a detonation driver is the key to creating high-quality driving flows.

\section{Detonation Driver with a Cavity Ring}

Although the numerical results presented in the preceding three cases show much improvement for driving flow uniformity, the postshock pressure behind the upstream-traveling shock wave is still lower than the $\mathrm{C}-\mathrm{J}$ pressure. To improve further the detonationdriver performance, type-C, configuration, as shown in Fig. 2, is studied in case 4 . The diameter ratio of the large-diameter tube to the cavity ring and to the small-diameter tube is taken to be $1.5: 2.5: 1.0$. It is expected that this special configuration could be useful to avoid the overdriven detonation, as well as to produce a stronger upstreamtraveling shock wave. Numerical results of this case are given in Figs. 9 and 10 .

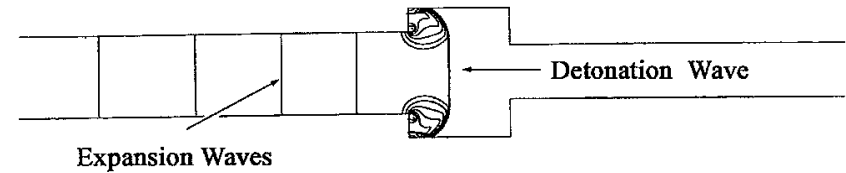

a)

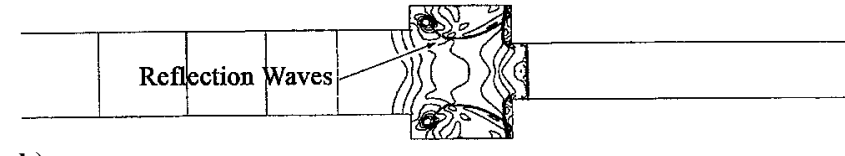

b)

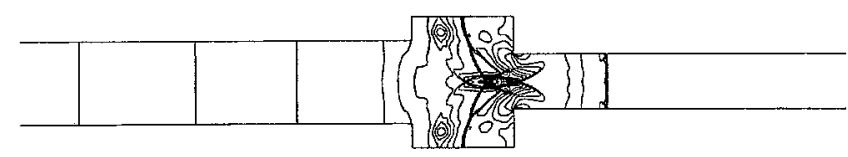

c)

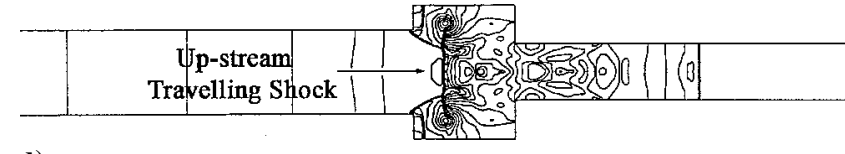

d)

Fig. 9 Time sequences of isobars of the flow motion showing wave interactions in case 4 with a cavity ring.

Sequential isobars of wave interactions near the cavity ring are shown in Fig. 9. Note that the detonation front first diffracts at the frontal surface of the cavity ring, as shown in Fig. 9a, and then reflects from its side wall, as seen in Fig. 9b. The diffracting detonation front bounces back later from the rear surface of the cavity ring, as shown in Fig. 9b. Then, the reflected shock waves coalesce toward the axis of symmetry and complex wave interactions develop in the cavity ring, as shown in Fig. 9c. A nearly normal shock wave is finally generated and traveling upstream, as shown in Fig. 9d.

Figure 10 shows four sequential pressure distributions along the axis of symmetry. The result in Fig. 10a corresponds to the flowfield in Fig. 9b at the time instant immediately after the detonation front moves out of the cavity ring. The pressure drop observable behind 

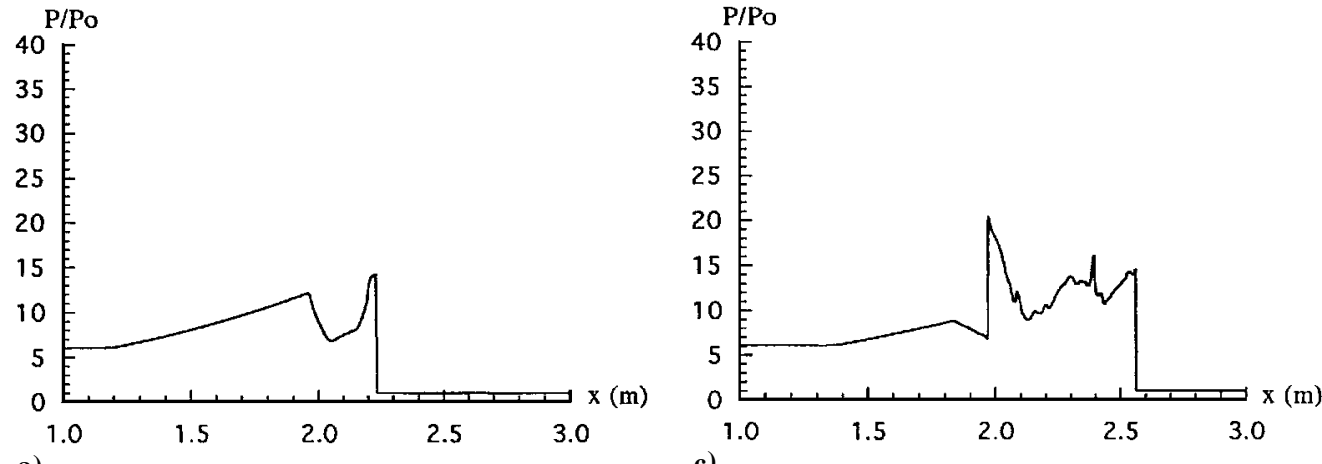

a)

c)
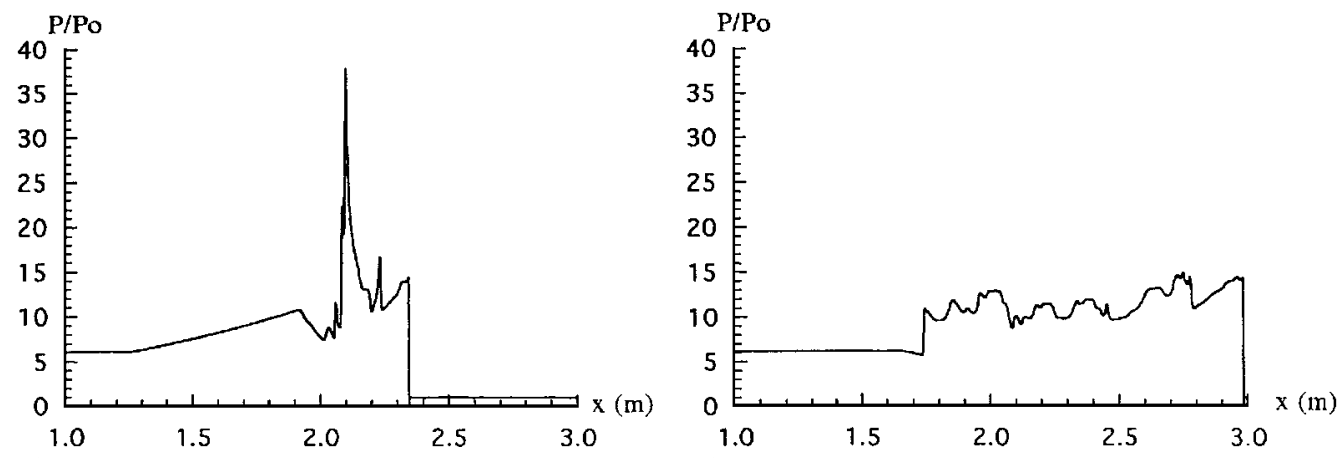

b)

Fig. 10 Sequential pressure profiles along the axis of symmetry of case 4 with a cavity ring.

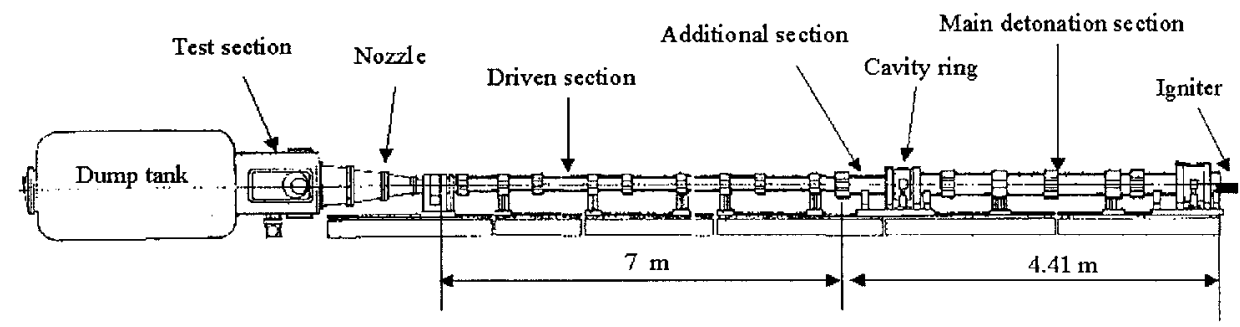

Fig. 11 Schematic of the new detonation-driven shock tunnel installed in Institute of Mechanics, Chinese Academy of Sciences, Beijing.

the detonation front is due to wave diffraction. Figure $10 \mathrm{~b}$ shows a pressure peak generated when the coalescence of reflected shock waves appears in Fig. 9c. The pressure peak decreases very rapidly, as seen in Fig. 10c, and the driving flow between the upstream traveling shock and the detonation front at almost uniform pressure is obtained (Fig. 10d).

When the pressure distribution shown in Fig. 10d is compared with those shown in Figs. 4d, 6d, and 8d, it is found that the induced pressure behind the upstream-traveling shock wave is higher, the detonation front is less overdriven, and the driving flow appears more uniform. This demonstrates that the configuration of case 4 could produce a much stronger upstream-traveling shock wave and avoid an overdriven detonation front. These are achieved due to the reflection of the diffracted detonation front from the rear surface of the cavity ring. Moreover, the flow energy stored in the cavity ring is also favorable for the generation of a more uniform driving flow.

\section{Experiments}

Based on the conclusion drawn from the numericalinvestigations, a new detonation-driven shock tunnel, as schematically shown in Fig. 11, is manufactured and installed at the Institute of Mechanics, Chinese Academy of Sciences, Beijing. This detonation-driven shock tunnel consists of six main parts: a main detonation section, a cavity ring, an additional detonation section, a driven section, a nozzle, and a dump tank. The main detonation section is $3.87 \mathrm{~m}$ long and $90 \mathrm{~mm}$ in diameter, the cavity ring is $360 \mathrm{~mm}$ long and $130 \mathrm{~mm}$ in diameter, and the additional detonation section is $180 \mathrm{~mm}$ long and $60 \mathrm{~mm}$ in diameter. The detonation driver, therefore, consists of these three sections. The driven section is $7 \mathrm{~m}$ long and $60 \mathrm{~mm}$ in diameter and is also referred to as the shock tube. The length of both the additional detonation section and the cavity ring are changeable so that the optimum configuration could be explored

Several experiments have been conducted by varying the initial pressure and mass fractions of the $\mathrm{H}_{2} / \mathrm{O}_{2}$ mixture. Stagnation pressure histories measured at the end wall of the driven section are presented to examine the uniformity of test flows. In the experiments, the shock tube is closed without a nozzle to remove the effects of the nozzle on the test gas flow. However, it is too difficult to measure the pressure profiles along the detonation driver and present them in the same manner as in the numerical simulations. Nevertheless, the stagnation pressure histories at the end wall of the shock tube could reflect the wave characteristics observed in the driving flows of the numerical simulations, for example, in Fig. 10d. The results from two experiments, as shown in Fig. 12, are obtained at the same initial condition of $P_{i}=0.5 \mathrm{MPa}, P_{i}=1.2 \mathrm{kPa}$, $\mathrm{H}_{2}: \mathrm{O}_{2}=4: 1$. Figure 12 shows that the two pressure profiles are similar to each other and that experimental repeatability is well demonstrated. Moreover, the two experimental results exhibit similar wave characters, as observed in the pressure profile in Fig. 10d: The pressure jump appears first, showing the arrival of the leading shock wave, followed by a pressure decrease, which is also observable in Fig. 10d. Then the second pressure jump comes due to the effect of the cavity ring and leads a uniform flow slug with pressure fluctuations, which attenuated much more than in Fig. 10d. Finally, another pressure jump appears, which is believed to be due to the reflected shock from the upstream closed end of the detonation driver. In addition, the reservoir pressure obtained with the detonation-driven shock tunnel could be maintained for as long as $4 \mathrm{~ms}$. When it is considered that the result is obtained with only a $4.41-\mathrm{m}-\mathrm{long}$ detonation driver, this driving time is extremely promising. 

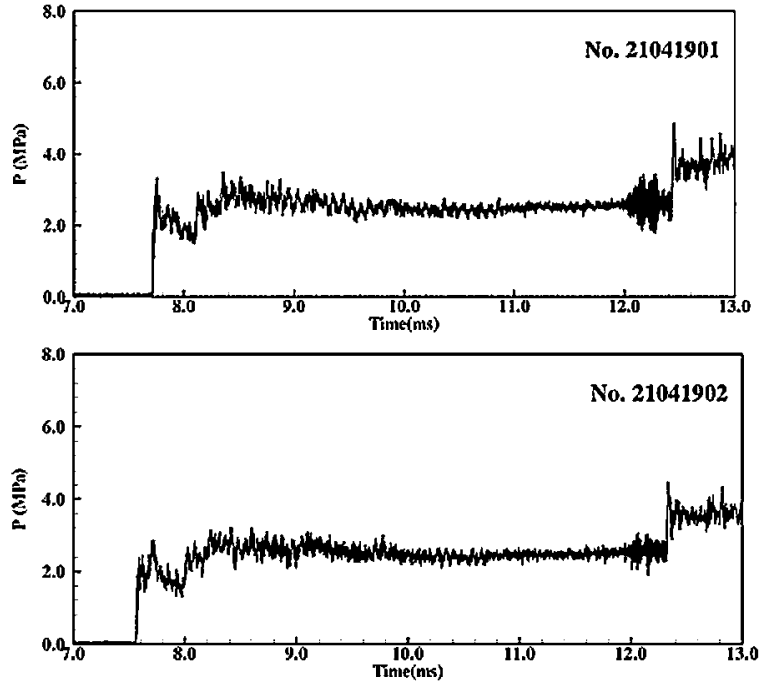

Fig. 12 Stagnation pressure histories measured at the end wall of the driven section in case 4 with a cavity ring from two experiments at the same conditions.

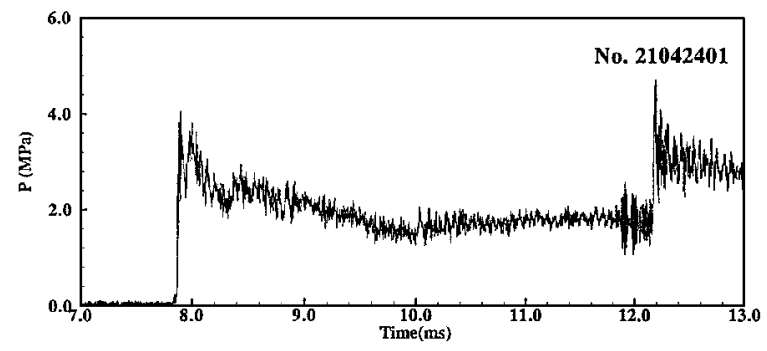

Fig. 13 Stagnation pressure histories measured at the end wall of the driven section in case 3 .

Stagnation pressure measured at the end wall of the driven section in case 3 is given in Fig. 13, and this case is the same as case 4 without any cavity ring. When this result is compared with Fig. 8d, similar features could be observed. At first, the arrival of the leading shock wave results in a pressure jump, then the pressure decrease follows, and finally a flow slug with an almost uniform pressure comes. Examination of Figs. 12 and 13, shows that the pressure jump behind the leading shock wave in Fig. 13 is higher than in Fig. 12, which is due to the effect of the overdriven detonation front, and as a reservoir gas flow, the flow state in Fig. 12 seems more preferable than that in Fig. 13.

These experimentsconfirm the conclusiondrawn from the numerical simulations, and the forward-running detonation driver with a cavity ring has demonstrated to be a more promising choice for high-enthalpy shock tunnels.

From the point view of shock wave dynamics, the wave phenomena examined in the forward-running detonation drivers described in this paper are summarized as follow: The detonation front is reflected at first in the area-changing section and then focuses on the axis of symmetry. This process results in an upstream-traveling shock wave and a downstream one. The upstream-traveling shock wave can increase the pressure of the flow it processes, and the downstream-traveling shock wave will lead to a overdriven detonation front. To improve flow uniformity in the driver, an overdriven detonation should be avoided, because otherwise it would be difficult for the upstream-traveling shock to induce a pressure level that is as high as the detonation pressure. Rather, it is more preferable to keep the pressure behind the detonation from increasing (as in an overdriven wave) to achieve a uniform driving flow. In the case of the detonation driver with a cavity ring, the upstream-traveling shock wave is stronger, and the downstream-travelingshock wave is weaker. Moreover, the energy stored in the cavity ring can compensate for the pressure decrease due to the Taylor expansion waves. This has also been demonstrated to be helpful for the generation of a more uniform driving flow. These three wave phenomena are the key points to consider for improving the driving flow in detonation drivers.

\section{Conclusions}

Various detonation drivers with the specially designed sections, operated in the forward-runningmodel, were numericallyexamined. Experiments were also conducted by using a new detonation-driven shock tunnel with a cavity ring. The results are summarized as follows: The reflection of detonation waves at the converging wall results in an upstream-traveling shock wave after complex wave interactions. This shock wave can increase the flow pressure that has decreased due to the trailing expansion waves. Avoiding an over-driven detonation front is also important for maintaining flow uniformity. Although the pressure fluctuationsdue to shock wave focusing and shock wave reflection are observable in these detonation drivers, they attenuate very rapidly as the detonation wave propagates downstream in the detonation driver and the leading shock in the shock tube. Moreover, the storage of part of the energy carried by detonation fronts in a cavity ring to compensate for the pressure decrease due to the Taylor expansion waves has demonstrated to be helpful for improving the driving flow. Among the tested detonation drivers, the configuration with a cavity ring is found to be most promising. Feasibility of the proposed detonation driver for high-enthalpy shock tunnels operating in a forward-running mode is well demonstrated.

\section{References}

${ }^{1}$ Stalker, R. J., "An Investigation of Free Piston Compression of Shock Tube Driver Gas," National Research Council, Mechanical Engineering Rept. MT-44, NRC, Ottawa, 1961.

${ }^{2}$ Stalker, R. J., "Study of the Free-Piston Shock Tunnel," AIAA Journal, Vol. 5, No. 12, 1967, pp. 2160-2165.

${ }^{3}$ Stalker, R. J., "Development of a Hypersonic Nozzle," Journal of the Royal Aeronautical Society, Vol. 76, 1972, pp. 376-384.

${ }^{4}$ Stalker, R. J., "Hypersonic Aerodynamics in Australia," Journal of the British Interplanetary Society, Vol. 41, No. 12, 1988, pp. 611-618.

${ }^{5}$ Hornung, H. G., "Performance Data of the New Free-Piston Shock Tunnel at GALCIT," AIAA Paper 92-3943, 1992.

${ }^{6}$ Eitelberg, G., McIntyre, T. J., Beck, W. H., and Lacey, J., "High Enthalpy Shock Tunnel in Göttingen, AIAA Paper 92-3955, 1992.

${ }^{7}$ Itoh, K., "Tuned Operation of a Free-Piston Shock Tunnel," Proceedings of the 20th International Symposium on Shock Waves, edited by B. Sturtevant, J. E. Shepherd, and H. G. Hornung, Vol. 1, World Scientific, Singapore, 1995, pp. 43-51.

${ }^{8}$ Itoh, K., Ueda, S., Komuro, T., Saito, K., Takahashi, M., Miyajima, H., and Koga, K., "Design and Construction of HIEST (High Enthalpy Shock Tunnel)," Proceedings of the International Conference on Fluid Engineering, Vol. 1, JSME Press, Tokyo, 1997, pp. 353-358.

${ }^{9}$ Bird, G. A., "A Note on Combustion Driven Shock Tubes," Rept. 146, AGARD, 1957

${ }^{10}$ Yu, H. R., "Shock Tunnel and Its Application to Aeroheating Experiments," Ph.D. Dissertation, Inst. of Mechanics, Chinese Academy of Sciences, July 1963 (in Chinese).

${ }^{11}$ Yu, H. R., "Experimental Study of Oxyhydrogen Detonation Driver for a Shock Tunnel," Proceedings of 1989 Japanese National Symposium on Shock Waves, 1989, pp. 1-7.

${ }^{12} \mathrm{Yu}$, H. R., "Oxyhydrogen Combustion and Detonation Driven Shock Tube," Acta Mechanica Sinica, Vol. 15, No. 2, 1999, pp. 97-107.

${ }^{13}$ Yu, H. R., Esser, B., Lenartz, M., and Grönig, H., "Gaseous Detonation Driver for a Shock Tunnel," Shock Waves, Vol. 2, No. 4, 1992, pp. 245-254.

${ }^{14}$ Lu, F. K., Wilson, D. R., Bakos, R. J., and Erdos, J. D., "Recent Advances in Detonation Techniques for High-Enthalpy Facilities," AIAA Journal, Vol. 38, No. 9, 2000, pp. 1676-1684.

${ }^{15}$ Chapman, D. L., "On the Rate of Explosions in Gases," Philosphical Magazine, Vol. 47, 1899, p. 90

${ }^{16}$ Jouguet, E., "Sur la Propagation des Reactions Chimiques dans les Gaz," Journal Maths Pure Appl, Vol. 7, 1905, p. 347.

${ }^{17}$ Taylor, G. I., "The Dynamics of the Combustion Products Behind Planar and Spherical Detonation Fronts in Explosive," Proceedings of the Royal Society of London, Series A: Mathematical and Physical Sciences, Vol. 200 1950, pp. 235-247.

${ }^{18}$ Jiang, Z. L., Falcovitz, J., and Takayama, K., "Numerical Simulations of Detonation in Converging Chambers," JSME International Journal, Vol. 40, No. 3, 1997, pp. 422-431.

${ }^{19}$ Jiang, Z. L., Takayama, K., and Chen, Y. S., "Dispersion Conditions for Non-Oscillatory Shock Capturing Schemes and Its Applications," Computational Fluid Dynamics Journal, Vol. 4, No. 2, 1995, pp. 137-150. 